\title{
Aproximaciones a los sentidos políticos de los estudios de recepción
}

Jorge Calles Santillana ${ }^{1}$

L os estudios de recepción han venido ganando aceptación en los campos de la comunicación social y la educación en los últimos años, modificando la concepción del proceso de la comunicación y sus implicaciones. El énfasis en las interpretaciones individuales y de grupo y la atención a los contextos en los que la recepción ocurre han puesto en claro que el estudio de la comunicación desde una perspectiva de la creación de significados ofrece una mirada de lo social diferente a la producida por la perspectiva de los efectos. Una de las principales implicaciones de la difusión de los estudios de recepción es que ha conducido a revalorar la comunicación de masas y la cultura mediática. Si el enfoque crítico de la Escuela de Frankfurt había devaluado la cultura de masas y la había definido como un ámbito de alienación, los estudios de recepción han mostrado que es un campo de luchas por la significación y un factor importante de la creación de identidades culturales. De esa forma, el carácter político de los estudios de recepción ha estado en debate a lo largo de estos años.

Este ensayo reúne algunas notas breves que me han permitido hacer una primera -y provisional- reflexión sobre el carácter político de tres líneas de estudio de la recepción. En primer lugar, me ocupo de los estudios de David Morley por haber sido uno de los pioneros en el campo y por haber afirmado el carácter político de los estudios de recepción. Reviso también las aportaciones de John Fiske y su creencia en la política de resistencia y liberación, surgida de los placeres productivos proporcionados por la cultura mediática. Finalmente, me ocupo de las aportaciones de Klaus Bruhn Jensen y Guillermo Orozco, quienes desde una perspectiva pragmática y pedagógica ofrecen elementos para considerar una política crítica que pueden conducir a la formación de culturas políticas democráticas radicales.

1. Universidad de la Américas, Puebla. Correo electrónico: jcalles@mail.udlap.mx. 


\section{La aportación de los estudios de recepción}

Los estudios de recepción surgen de una doble ruptura crítica y han creado un espacio en el cual las relaciones individuo-sociedad y estructura-acción social han podido ser repensadas desde el campo de la comunicación. Por una parte, los estudios de la recepción retomaron el aporte del enfoque de usos y gratificaciones, lo despojaron de sus raíces psicologistas e individualistas y le otorgaron un sentido crítico-social. Por otra parte, movieron el análisis ideológico más allá del texto e hicieron evidente que el proceso de creación de hegemonía es más complejo y contradictorio que lo que los primeros estudios críticos habían sugerido.

El objeto de estudio que surge de tal ruptura es un fenómeno social que, tanto en los primeros estudios sobre los medios de comunicación como en los análisis marxistas de la ideología, era considerado el acto final y la consecuencia última del proceso de la comunicación masiva: el acto de seleccionar, exponerse a y apropiarse de los productos de las industrias culturales. Para los estudios de recepción, el consumo deja de ser un mero acto individual de exposición en el que o bien la psique humana se ve afectada por el producto ofrecido por el medio o la poco explorada alienación de las conciencias sociales se da por confirmada. La recepción empieza a ser considerada, entonces, como el acto en el cual el encuentro entre los textos mediáticos y las subjetividades sociales genera procesos de significación que pueden tener múltiples orientaciones e implicaciones.

La reconceptualización del proceso de recepción acarreó, por un lado, la revisión crítica del concepto de audiencias acuñado por los estudios positivistas de la tradición de los efectos y que fue preservado por los estudios de usos y gratificaciones. Significó, también, la introducción de la noción en el discurso crítico. El carácter superestructural del que originalmente fue dotado el concepto de ideología convertía en teoréticamente irrelevante el proceso de recepción en la tradición marxista. En línea con la teoría de la producción económica, el acto de recepción se entendía como un mero acto de consumo en el que el capital se realizaba. No obstante, los nuevos estudios de recepción quedaron inscritos dentro de la línea de los estudios críticos, aun cuando en el paradigma de los efectos existía una larga tradición de investigación sobre la materia. De esa manera, los estudios de la recepción conservaron el interés por el consumo presente en los análisis de efectos, pero definieron el fenómeno como una práctica social en la que múltiples fuerzas sociales le dan forma y sentido.

El carácter industrial de los productos de los medios es una de esas fuerzas que influyen en el proceso de recepción. Las industrias culturales, al igual que cualquiera otra industria capitalista, producen masivamente para 
una audiencia también masiva siguiendo fórmulas, códigos y normas convencionales (Kellner, 1995: 1). La cultura ofrecida por esta industria es comercial y sus productos son mercancías cuyo fin es crear una audiencia y convertirla, a su vez, en una mercancía para las industrias ávidas de publicidad. En las sociedades contemporáneas, la cultura mediática dominante se convierte en una fuente muy importante de pedagogía cultural: contribuye a educar a las audiencias sobre cómo comportarse y qué pensar, sentir, temer, desear, aceptar y rechazar (Kellner, 1995: 2).

Sin embargo, los estudios de recepción están informados por una lectura crítica del concepto de ideología y, por tanto, rechazan que el proceso de recepción implique una forzosa aceptación y reproducción de los valores ideológicos presentes en los productos culturales. Stuart Hall, siguiendo a Althusser, plantea que a la ideología hay que entenderla como sistemas de representación a través de los cuales hombres y mujeres viven. Esto es, la ideología no es un sistema cerrado de significación, sino un conjunto de cadenas discursivas, representaciones, campos semánticos y formaciones discursivas (1991: 102; 1997: 16-17). El concepto de polisemia ${ }^{2}$ se ha convertido, por tanto, en el eje central de los estudios de recepción (Fiske, 1987; Jensen, 1995; Morley, 1992) y ha dado pie para establecer diferencias entre la economía financiera (en la que circulan bienes, servicios y recursos económicos) y la economía cultural (en la que circulan signos y significados) (Fiske, 1987). $\mathrm{El}$ objetivo, entonces, de los estudios de recepción es investigar cómo esas diferentes cadenas de significación son apropiadas y cuáles significados son recreados e introducidos en prácticas cotidianas por quienes las reciben.

El contexto en el cual ocurre el proceso de recepción ha sido otro elemento importante que la investigación culturalista ha enfatizado (Morley, 1992; Turner, 1990). Los estudios sobre efectos jamás se ocuparon de analizar el lugar en el que el proceso de recepción tiene lugar, ni los factores sociales y culturales que lo influyen. Preocupados fundamentalmente por encontrar patrones generales que afectan las preferencias de exposición y uso, los estudios positivistas reúnen grandes números de sujetos para interrogarlos sobre sus particulares hábitos y actitudes e ignoran las condiciones concretas en las que la recepción tiene lugar. Para los estudios culturalistas, en cambio, el contexto socio-histórico de la recepción es parte del proceso mismo y la investigación no puede excluirlo del análisis. De esa forma, la investigación de la recepción ha contribuido a percibir y estudiar la comunicación como un proceso de creación de significados y no como un proceso de transmisión de información, concepción que se desprende del paradigma positivista del estudio de los efectos (Carey, 1991; Orozco, 1987).

2. Por polisemia se entiende los múltiples significados que los signos pueden adquirir (Fiske, 1987: 15). 
Si bien todas estas consideraciones han contribuido a darle forma a los estudios de recepción, el problema teórico central del esfuerzo ha sido el de la definición del concepto mismo de audiencia. Extraída de los estudios de efectos, la noción de audiencia en los estudios culturalistas se presentaba como un concepto que posibilitaría el avance en los estudios sobre ideología y control social. Sin embargo, sus raíces positivistas darían pauta también para poner en entredicho su potencial. Desde los primeros estudios de recepción, la noción fue cuestionada, lo mismo que los métodos por medio de los cuales fue construida como objeto de estudio y analizada (Allor, 1988). Actualmente el debate continúa, al grado que la relevancia de los estudios de recepción ha sido considerada dudosa (Huertas, 2001; Nightingale, 1996). La discusión es de primera importancia debido a que pone sobre la mesa no sólo el sentido crítico de los estudios de recepción sino, y sobre todo, su carácter político.

\section{El sentido político de los estudios de recepción}

En un ensayo publicado originalmente en 1989, Ien Ang (1996) revisó las aportaciones de los estudios de audiencia de David Morley destacando que el enfoque etnográfico adoptado por el investigador británico representaba un significativo avance en el estudio de la recepción. Según Ang, aun cuando el trabajo de Morley resaltaba el trabajo productivo de los receptores, su propuesta no coincidía con la de las teorías de los usos y gratificaciones, estudios que representaban en ese momento la posición más avanzada dentro del paradigma de los efectos. Informados por teorías psicologistas y estructuralfuncionalistas, los estudios de usos y gratificaciones teorizaban a los receptores como individuos activos que seleccionaban racionalmente los productos mediáticos que mejor satisficieran sus necesidades psicológicas. En el trabajo de Morley, en cambio, Ang encontraba una intencionalidad política puesto que su objetivo no era describir la actividad de la audiencia désde una perspectiva meramente individual, sino el de hacer visibles los procesos estructurales y culturales a través de los cuales la audiencia es constituida como tal.

Un punto teórico esencial del enfoque de los estudios culturales de audiencia es destacar que las dinámicas de ver televisión, sin importar cuán heterogéneas y aparentemente libres sean, están siempre relacionadas con las operaciones de las formas del poder social (1996: 42. Traducción mía).

Así pues, marcando una clara distinción con el enfoque de usos y gratificaciones, los analistas culturalistas mostraban su interés por orientar dentro de la tradición crítica sus estudios empíricos. 
En su estudio de Nationwide, un programa de revista británico transmitido nacionalmente a fines de los años setenta y principios de los ochenta, Morley (1980) puso a prueba el modelo de encodificación/decodificación que Stuart Hall había desarrollado a fines de la década de los setenta, tratando de identificar los procesos de apropiación de los contenidos ideológicos del programa por varios receptores pertenecientes a diferentes grupos y clases sociales. Morley encontró que los sujetos de su investigación establecían procesos de negociación con las propuestas ideológicas del programa en una forma que conducía a repensar el concepto de clase del marxismo y que daba sustento a la propuesta de Hall y sus presupuestos althusserianos acerca de la autonomía de la esfera cultural (Morley, 1992: 12-13). Desarrollos posteriores de su trabajo conducirían a Morley a sostener que el carácter político de la investigación de audiencias radicaba en las posibilidades de analizar en los contextos de recepción los procesos por medio de los cuales los niveles micro y macro sociales confluían, al igual que lo público y lo privado. La idea central era analizar y entender "los placeres que la cultura popular ofrece a sus consumidores" para comprender cabalmente "cómo opera la hegemonía a través de los procesos de cultura popular” (1992: 36. Traducción mía). El contexto micro de la recepción, proponía Morley, permitía el análisis de las fuerzas macro que le daban forma a la sociedad y se convertían en valores hegemónicos de los cuales los sujetos subordinados extraían placer.

Aun cuando el trabajo de Morley es etnográfico, su enfoque es también sociológico y teóricamente más inclinado al estudio de las fuerzas estructurales que ejercen influencia sobre la recepción. Por lo tanto, el sentido político de su trabajo tiene un carácter de denuncia. Mediante el estudio de los contextos de recepción, Morley intentaba exponer los diferentes procesos por medio de los cuales fuerzas estructurales tales como la clase, la disponibilidad de tecnologías de comunicación en el hogar, los roles sociales asignados a los géneros sexuales y el poder socialmente construido en el seno del hogar, influenciaban el uso de los medios y las interpretaciones de sus textos. Asimismo, estudiaba la forma en la cual las nociones de lo local, lo nacional y lo internacional eran percibidas y construidas desde el microcosmos del hogar, dependiendo de género sexual y edad (Morley, 1992: 276-278). No es accidente, por tanto, que en el trabajo de Morley no se encuentren estudios sobre prácticas de oposición y resistencia a productos mediáticos específicos.

Una propuesta política diferente, que podríamos llamar de resistencia, es identificable en el trabajo de otros autores. Janice Radway (1984), por ejemplo, estudió por qué mujeres de la India eran asiduas lectoras de novelas románticas durante sus tiempos libres. La investigadora se ocupó de estas prácticas debido a que le resultaba difícil de entender que novelas de escaso valor literario pudieran ser atractivas y útiles a sus lectoras. Radway encontró que las mujeres realizaban la lectura en privado, como una forma de estable- 
cer distancia respecto de las múltiples labores domésticas que estaban bajo su responsabilidad. Así, la lectura de novelas románticas representaba para las mujeres indias una especie de:

[...] tácita y mínima protesta contra la constitución patriarcal de las mujeres. La lectura les permite demarcar un espacio en el cual las mujeres pueden dejar de lado el desinterés que demandan sus labores (Radway, 1984: 233 traducción mía).

Radway encontró que el placer proporcionado por la lectura permitía a las mujeres indias sobreponerse del cansancio y el sinsentido de las labores domésticas que el sistema patriarcal les imponía. Mientras los estudios de Morley muestran la confluencia de fuerzas estructurales y placeres domésticos, Radway enfatiza el carácter reivindicativo de la lectura privada. El placer aparece aquí como una actividad no necesariamente de resistencia, pero sí de reafirmación. Las mujeres se aíslan no sólo para escapar de la pesada carga del trabajo doméstico, sino también para vivir fantasías amorosas que supuestamente deberían ser realidades.

Sin embargo, es sin duda el trabajo de John Fiske el que mejor representa la línea política de resistencia de los estudios de recepción. En palabras de Nicholas Abercrombie y Brian Longhurst, en un continuo cuyos polos fueran el Texto Dominante y la Audiencia Dominante, el trabajo de Fiske quedaría ubicado claramente en este segundo extremo (1998: 23).

John Fiske es quizás el autor que más se ha ocupado de teorizar el carácter activo de las audiencias. Contrariamente a la propuesta de la revista Screen, según la cual las audiencias eran entendidas como sujetos textuales, Fiske sostiene el carácter social de los receptores de los medios. Los sujetos sociales, plantea Fiske:

[...] tienen historia, viven en formaciones sociales particulares (una combinación de clase, género, edad, región, etc.) y están constituidos por una historia cultural compleja que es tanto social como textual. La subjetividad se forma a través de experiencias sociales "reales" y experiencias mediadas o textuales (Fiske, 1987: 62).

Las audiencias están compuestas de sujetos sociales cuyas experiencias sociales son más importantes e influyentes en la construcción de significados que las experiencias textuales. De esa forma, Fiske enfatiza el carácter activo de las audiencias.

La creación de significados que resulta del proceso de recepción no puede ser entendida en esta perspectiva como una simple reproducción de los significados propuestos en los textos por sus productores, sino un diálogo entre el texto y el sujeto social (Fiske, 1987: 66). Si se admite que los textos mediáticos, especialmente el televisivo, tienen que ser polisémicos para poder 
alcanzar a una gran diversidad de grupos sociales y ofrecerles placer, y que tanto los textos mediáticos como las subjetividades sociales son construcciones discursivas, se entiende que de las coincidencias y contradicciones existentes entre ambas formaciones discursivas surjan multiplicidad de lecturas.

Este desarrollo teórico ha conducido a Fiske a realizar estudios de recepción de los cuales puede extraerse una política de la recepción diferente a la de los estudios revisados anteriormente. Para Fiske, la cultura mediática (o popular culture como es nombrada por un buen número de críticos en el mundo anglosajón) es por definición política porque es "producida y disfrutada bajo condiciones de subordinación social y está íntimamente ligada en las relaciones de poder sociales" (1992: 159. Traducción mía). La cultura popular, argumenta Fiske, trabaja fundamentalmente en el campo de la oposición popular más que en el de la oposición radical. Su carácter político es, por tanto, progresivo, no radical. Sin embargo, esta política progresiva es una condición necesaria para la política radical. La cultura popular, plantea Fiske, "no sólo mantiene las diferencias sociales sino también su oposición y la conciencia de la gente de tales diferencias" (1992, 161. Traducción mía).

El carácter político progresivo de la cultura popular posibilita que la gente, bajo determinadas circunstancias y condiciones, adquiera poder y pueda actuar especialmente en el micronivel, incrementando su espacio sociocultural y generando una micro-redistribución del poder a su favor. Contrariamente a los estudios críticos que a partir de la Escuela de Frankfurt han enfatizado el carácter industrial y alienante de la cultura de medios, Fiske enfatiza la capacidad creativa de los receptores. Para él, la cultura popular es hecha por la gente y no producida por las industrias culturales (1992: 24). El planteamiento implica, por tanto, la deconstrucción de las tradicionales categorías sociológicas y la afirmación de las identidades culturales. Asimismo, implica ver la vida cotidiana no como un área de permanente sometimiento y alienación, sino como un campo en el que las relaciones de poder son permanentemente cuestionadas vía lo que Fiske llama los placeres productivos $(1987,1992)$.

Aun cuando el trabajo de Fiske es mayoritariamente teórico, algunos de sus trabajos dan cuenta de cómo algunos grupos culturales emplean productos de las industrias culturales para reconocerse como fuerzas opositoras y reafirmar sus posiciones críticas hacia fuerzas ideológicas dominantes. Sus estudios sobre la apropiación crítica de la imagen de Madonna (1990) por parte de muchachas adolescentes y sobre la fascinación de un grupo de vagabundos despertada por la película Duro de matar (1996) para expresar su rechazo al sistema social que los ha estigmatizado como perdedores y carentes de valor, son especialmente importantes.

Dos puntos son relevantes en el trabajo de Fiske. Primero, destaca el carácter político de la vida cotidiana y, bajo una clara influencia foucaultiana, 
le confiere gran importancia a los micro procesos a través de los cuales el poder social se establece y es contestado. Segundo, resalta la capacidad crítica de los actores sociales y los procesos por medio de los cuales los sujetos sociales fijan, negocian y redefinen permanentemente sus identidades. No obstante, habrá que reconocer que la mayoría de sus críticos aciertan cuando afirman que Fiske posee un exagerado optimismo que casi termina siendo una apología de la cultura mediática comercial.

Mientras Morley, Ang y otros autores persiguen hacer visibles las estructuras de poder que afectan a los procesos de consumo cultural, Fiske se ocupa de destacar la capacidad contestataria de los consumidores y el potencial político de los consumos placenteros productivos. Frente a la propuesta de Fiske, la política denunciante de los primeros estudios de audiencias parece resultar descriptiva y poco útil. Si bien ambos enfoques coinciden en que la recepción sólo se torna en un objeto de estudio legítimo cuando se le analiza desde la perspectiva del poder, la propuesta de Fiske se muestra más ambiciosa porque su análisis es dinámico y se ocupa de prácticas constitutivas de poderes y contrapoderes.

No obstante, la propuesta de Fiske da por hecho que el poder es siempre contestado en todo lugar, y que el simple hecho de consumir convierte a las audiencias en sujetos políticos con poder y capacidad para resquebrajar el orden social en cualquier momento y a cualquier nivel. Si Morley y Ang privilegian el poder de las estructuras, Fiske exagera la acción social.

Una tercera propuesta política es identificable en los trabajos de Klaus Bruhn Jensen y Guillermo Orozco. El primero sugiere prestar más atención al análisis de la polisemia de la recepción que a la de los mensajes (1995). El segundo, por su parte, enfatiza el carácter social de la práctica de la recepción y destaca que los factores estructurales que tienden a imprimir significados ideológicos a los mensajes pueden ser deconstruidòs y resignificados por los receptores (1990).

Basado en una perspectiva pragmática, Jensen propone una semiótica social que se ocupe de estudiar el uso social de los signos $(1995,1996)$. En línea con el pensamiento de Charles S. Pierce, Jensen sostiene que los signos no son lo que conocemos sino el cómo llegamos a conocer lo que podemos justificar diciendo que sabemos. Es a través de signos que la gente interactúa con una realidad de diversos objetos, eventos y discursos (Jensen, 1996: 69). Jensen retoma el carácter relacional que el significado adquiere en la semiótica de Pierce para proponer el estudio semiótico social de la comunicación. El eje del análisis es el estudio de la producción de significado en su contexto social. Cuando los contenidos de los medios son interpretados y apropiados por los actores sociales y se constituyen en la base de su conocimiento y acción, se puede decir que los discursos de los medios están produciendo efectos sociales (1996: 70). 
Las implicaciones de esta propuesta para el estudio de la recepción son varias. En primer lugar, lleva a considerar a las audiencias de los medios no sólo como conjuntos de individuos definidos por sus roles sociales y sus características demográficas, sino -y sobre todo- por sus marcos interpretativos o repertorios con los cuales se apropian de los contenidos de los medios y de otros productos culturales (Jensen, 1995: 12). En segundo lugar, orienta el estudio de la recepción hacia la relación entre las estructuras macrosociales y los micro procesos de recepción (Jensen, 1996: 71). La relevancia de la aportación de Jensen radica en que pone de manifiesto que el mundo es aprehendido a través de conocimiento adquirido en la práctica a través de signos. En la medida en la que los medios de comunicación son una de las fuentes principales de signos en la vida contemporánea y en la que el conocimiento es una práctica contextual, la propuesta de Jensen sugiere abordar la recepción como una práctica desde la cual se puede generar conocimiento nuevo y distinto desde el cual el mundo pueda ser re-aprehendido y, consiguientemente, transformado.

Por su parte, Guillermo Orozco (1990) propone una pedagogía de medios crítica, que aun cuando fue diseñada originalmente para promover el alfabetismo mediático entre los niños, puede retomarse para una propuesta más amplia. Fundamentado en la perspectiva teórica del aprendizaje social, Orozco propone que los receptores de los medios son aprendices sociales cognoscitivamente activos (1990: 38-39) y que el aprendizaje ocurre en contextos históricos y socioculturales específicos que inciden en él. Según Orozco, el aprendizaje se produce a través de una doble mediación: cognoscitiva y sociocultural. Orozco emplea el concepto de guion, sugerido por Durkin, para explicar esta doble mediación. Un guion es entendido como la representación mental de una secuencia de eventos ordenados de acuerdo con una intencionalidad y con el propósito de alcanzar una meta (1990: 40). El conocimiento de la realidad que adquirimos socialmente se construye a través de los múltiples guiones que las instituciones sociales - incluidos los medios- nos proporcionan de manera permanente. La recepción de productos de medios puede ser entendida como una práctica en la que los actores sociales son invitados a reforzar y/o a modificar tales guiones.

La propuesta cognoscitiva de Orozco y la propuesta semiótica de Jensen apuntan hacia un proyecto de recepción que sugiere una práctica de identificación de los procesos y los mecanismos a través de los cuales se generan las significaciones sociales y la posibilidad de generar nuevas prácticas cognoscitivas.

Varias son las diferencias entre las implicaciones políticas de esta propuesta y las de las propuestas discutidas anteriormente. En primer lugar, la actividad de las audiencias deja de ser únicamente un objeto de estudio susceptible de explicación y se convierte en praxis transformadora. De esta forma, la recepción puede ser definida como el punto de llegada de un 
proyecto político de pedagogía mediática emancipadora y, al mismo tienpo, como el punto de partida de un proyecto de creación de una cultura política democrática radical (Calles, 1999). ${ }^{3}$

En segundo lugar, esta propuesta establece que las fuerzas estructurales que cercan ideológicamente las significaciones propuestas por los productos de las industrias culturales no pueden ser analizadas independientemente de la actividad desarrollada por los consumidores en el momento de la recepción. La recepción es un acto semiótico social en el que tanto las fuerzas estructurales como los placeres y las motivaciones de los actores sociales se conjugan para crear significados. No es, pues, sino a través del concepto de práctica social que el fenómeno de la recepción puede ser estudiado y convertido en práctica política. Las fuerzas estructurales ni son todopoderosas, ni actúan por sí mismas. Al mismo tiempo, la actividad de los actores sociales no es una práctica que tienda a conferirles poder necesariamente. En qué circunstancias y bajo qué condiciones las estructuras afectan de manera más efectiva a la recepción y en qué momentos y mediante qué mecanismos el consumo se convierte en práctica emancipadora, son algunas de las interrogantes que los estudios de la recepción tienen que ilustrar.

En tercer lugar, esta propuesta exige a los analistas poner atención a todo tipo de procesos de recepción y no sólo a aquellos en los que prevalecen la afirmación de identidades de oposición y de prácticas de resistencia.

Finalmente, la propuesta reclama un profundo ejercicio de reflexividad. Si el interés político de la propuesta es transformar los procesos semióticos mediante los cuales las audiencias producen y reproducen guiones de interpretación de la realidad, el sentido de la transformación se convierte, en sí mismo, en objeto de reflexión política. Es un proyecto que exige responder por qué es necesario deconstruir los procesos sociales de significación y justificar el sentido y la orientación de los nuevos cánones de creación de significado.

3. La teoría de la democracia radical, planteada por Chantal Mouffe (1992), busca sintetizar los planteamientos individualistas de la teoría liberal de la democracia y los del bien común propuestos por las teorías del republicanismo cívico. Para esta autora, la actividad político-social debe perseguir, al mismo tiempo, la creación de beneficios colectivos pero no el sacrificio individual. De allí que la sociedad no pueda ser organizada alrededor de una única idea sustantiva del bien común. El proyecto de la democracia radical requiere, por tanto, que los actores sociales sean capaces de crear una cadena de equivalencias entre luchas democráticas. Requiere, asimismo, la creación de una identidad política entre actores democráticos. 


\section{Conclusión}

Uno de los debates más importantes acerca de los estudios de la recepción ha sido el de su sentido y aportaciones políticas. Mientras algunos críticos han considerado que el énfasis en los consumos, interpretaciones y usos de los productos de los medios ha acarreado la pérdida del sentido crítico de los estudios de comunicación, otros en cambio han sostenido que el estudio de la recepción ha contribuido a entender de manera más profunda el proceso de creación -de hegemonía; otros más, inclusive, han sugerido que desde la recepción se pueden iniciar la ruptura de las significaciones hegemónícas. En este ensayo he revisado algunas de estas posiciones y he enfatizado que las aportaciones de Jensen y Orozco pueden ser útiles para emprender un proyecto político desde la recepción. Su objetivo sería, en primer lugar, generar una práctica pedagógica que condujera a los receptores a reconocer las representaciones y las significaciones hegemónicas y, en segundo término, propiciar la creación de culturas políticas democráticas radicales a partir de las cuales podrían planearse, organizarse y llevarse a la práctica transformaciones políticas de las estructuras sociales.

\section{Bibliografía}

Abercrombie, N. y B. Longhurst (1998), Audiences, Londres, Sage.

Allor, M. (1988), "Relocating the site of the audience", en Critical Studies in Mass Communication, septiembre, pp. 217-233.

Ang, I. (1996), Living room Wars, Londres, Routledge.

Calles, J. (1999), "Recepción, cultura política y democracia”, en Comunicación y Sociedad, núm. 36, pp. 47-70.

Carey, J. (1991), Culture as Communication, Londres, Unwin Hyman.

Fiske, J. (1996), "Audiencing Violence: Watching Homeless Men Watch Die Hard", en J. Hay et al. (eds.), The Audience and its Landscape, Boulder, Westview, pp. 297-316.

- (1992), Understanding Popular Culture, Londres, Routledge.

- (1990), Reading the Popular, Boston, Unwin Hyman.

- (1987), Television Culture, Londres, Routledge.

Hall, S. (1997), "The Work of Representation", en S. Hall (ed.), Representation, Londres, Sage, pp. 13-74.

_- (1991), "Signification, representation, ideology: Althusser and the post- 
structuralist debates", en Robert K. Avery y David Eason (eds.), Critical Perspectives on Media and Society, Nueva York, Guilford, pp. 88-113.

Huertas, A. (2001), La audiencia investigada, Barcelona, Gedisa.

Jensen, K. B. (1996), "After convergence: Constituents of a Social Semiotics of Mass Media Reception", en J. Hay et al. (eds.), The Audience and its Landscape, Boulder, Westview, pp. 63-74.

- (1995), The Social Semiotics of Mass Communication, Londres, Sage.

Kellner, D. (1995), Media Culture, Londres, Routledge.

Morley, D. (1992), Television, Audiences \& Cultural Studies, Londres: Routledge.

(1980), The "Nationwide" audience, Londres, British Film Institute.

Mouffe, C. (1992), "Democratic Citizenship and the Political Community", en C. Mouffe (ed.), Dimensions of Radical Democracy, Londres, pp. 57-102.

Nightingale, V. (1996), Studying Audience, Londres, Routledge.

Orozco, G. (1990), "El niño como televidente no nace, se hace", en M. Charles y G. Orozco (eds.), Educación para la recepción, México, DF, Trillas, pp. 33-48.

- (1987), Televisión y producción de significados: tres ensayos, Guadalajara, México, UdeG.

Radway, J. (1984), Reading the Romance, Chapel Hill, University of North Carolina Press.

Turner, G. (1990), British Cultural Studies, Londres, Unwin Hyman. 